may accelerate or exacerbate disease pathogenesis ${ }^{1} . \beta$-wrapin proteins were engineered to bind and sequester amyloidogenic monomers, and thus prohibit amyloid formation. $\mathrm{ZA} \beta_{3}$, the first reported $\beta$-wrapin ${ }^{2}$ sequesters a $\beta$-hairpin conformation of $\mathrm{A} \beta$, prohibiting aggregation of $\mathrm{A} \beta$ monomers into toxic forms ${ }^{3}$. $\beta$-wrapin variants have been engineered with varying activities for $\alpha$-syn and $\mathrm{IAPP}^{4-6}$, and ZSYM73, a $\beta$-wrapin with a $\mathrm{pM}$ affinity to $\mathrm{A} \beta^{7}$. Methods: Molecular dynamics simulations, free energy calculations, and surface plasmon resonance, among others enable us to uncover the binding and specificity of $\beta$-wrapins' for the three amyloidogenic proteins ${ }^{8}$. Results: Our studies reveal the key interactions acting as potential switches diminishing $\beta$-wrapins' affinity for $\mathrm{A} \beta$ / $\alpha$-syn, and suggest that IAPP is a comparatively promiscuous $\beta$ wrapin target. We delineate the distinct role of energetic driving determinants leading to $\beta$-wrapin binding and specificity; while both nonpolar and polar interactions synergistically contribute to binding, the polar binding energy component is the key energetic determinant contributing to $\beta$-wrapins' high-affinity. In line with this, our studies show that the high-affinity of ZSYM73 ${ }^{7}$ is attributed to salt-bridges stabilizing the ZSYM73:A $\beta$ complex. Additionally we show that multi-targeted binding properties of $\beta$-wrapins originate mainly from optimized interactions between $\beta$-wrapin residues and sets of residues in the three amyloidogenic proteins with similar physicochemical properties. Conclusions: Computational tools allow us to predict $\beta$-wrapin affinity for amyloidogenic proteins. We are currently using the insights from our studies to design new $\beta$-wrapins with improved affinities for one or combinations of the amyloidogenic proteins, which may constitute a promising and efficient direction for the future treatment of Alzheimer's disease in place of molecules binding to $\mathrm{A} \beta$ only. 1 . Luo J et al. J Biol Chem. 2016;291(32):16485-93. 2. Luheshi LM et al. PLoS Biol. 2007;8(3): e1000334. 3. Hoyer W, Härd T. J Mol Biol. 2008;378:398-411. 4. Mirecka EA et al. J Biotechnol. 2014;191:221-7. 5. Shaykhalishahi Het al. Chembiochem. 2015;16:411-414. 6. Mirecka EA et al. Angew Chem Int Ed Engl. 2014;53:4227-4230. 7. Lindberg H et al. Biotechnol J. 2015;10(11):1707-18. 8. Orr AA et al. J Phys Chem B. 2016;120(50):12781-12794.

\section{O2-02-05 RNA-BINDING PROTEINS WITH MIXED CHARGE DOMAINS SELF-ASSEMBLE AND AGGREGATE IN ALZHEIMER'S DISEASE}

Isaac J. Bishof ${ }^{1}$, Eric B. Dammer ${ }^{1}$, Duc Duong ${ }^{1}$, Marla Gearing ${ }^{1}$, James J. Lah ${ }^{2}$, Allan I. Levey ${ }^{2}$, Nicholas T. Seyfried ${ }^{1},{ }^{1}$ Emory University, Atlanta, GA, USA; ${ }^{2}$ Emory University School of Medicine, Atlanta, GA, USA. Contact e-mail: ibishof@emory.edu

Background: U1 small nuclear ribonucleoprotein $70 \mathrm{kDa}$ (U1-70K) and other RNA binding proteins (RBPs) are mislocalized to cytoplasmic neurofibrillary Tau aggregates in Alzheimer's disease (AD), yet understanding of the mechanisms that cause their aggregation is limited. Many of the RBPs that aggregate in neurodegenerative diseases self-assemble into RNA granules through low complexity (LC) domains. The intrinsically disordered C-terminus of U1-70K harbors a unique mixed charge LC domain, comprised of complementary arrays of basic $(\mathrm{R} / \mathrm{K})$ and acidic $(\mathrm{D} / \mathrm{E})$ residues, that we hypothesize are necessary for U1-70K aggregation and association with Tau in AD brain. Methods: To analyze the functional roles of the U1-70K LC domains, we performed co-immunoprecipitation and quantitative mass spectrometry analysis of recombinant U1-70K and deletions lacking the C-terminal LC domain(s). Immu- nocytochemistry was used to localize various recombinant U1-70K mutants and mixed charge proteins. We performed post-hoc analysis of a previously published insoluble $\mathrm{AD}$ brain proteome to assess the enrichment of structurally similar RBPs in AD brain tissue. In addition, immunoprecipitation of recombinant U1-70K fragments from human $\mathrm{AD}$ brain homogenate was performed to assess interactions with pathological tau. Results: A network-driven approach resolved functional classes of U1-70K interacting proteins that showed dependency on the U1-70K LC domain(s) for their interaction. This included structurally similar RBPs, such as LUC7L3 and RBM25, which require their respective mixed charge LC domains for reciprocal interactions with U1-70K and for participation in nuclear RNA granules. A significant proportion of RBPs with mixed charge domains have elevated insolubility in the AD brain proteome compared to controls. Furthermore, we show that the mixed charge LC domain of U1-70K can interact with Tau from AD brain. Conclusions: These findings highlight potential mechanisms for mixed charge domains in stabilizing RBP interactions and in mediating aggregation with pathological Tau isoforms in $\mathrm{AD}$.

\section{O2-02-06 PROPAGATION AND DIVERSE EFFECTS OF DISEASE-SPECIFIC PRION-LIKE TAU OLIGOMERIC STRAINS}

Julia E. Gerson ${ }^{1}$, Kathleen Farmer ${ }^{2}$, Urmi Sengupta ${ }^{2}$, Mariana Carretero-Murillo ${ }^{2}$, Salome McAllen ${ }^{2}$, Nemil Bhatt ${ }^{2}$, Anna Ellsworth ${ }^{2}$, Yonju $\mathrm{Ha}^{2}$, Wenbo Zhang ${ }^{2}$, Rakez Kayed ${ }^{2}$, ${ }^{1}$ University of Michigan, Ann Arbor, MI, USA ${ }^{2}$ University of Texas Medical Branch, Galveston, TX, USA.

Contacte-mail: rakayed@UTMB.EDU

Background:Tau aggregation is a common factor in many different neurodegenerative diseases, known as tauopathies. However, each tauopathy is characterized by a unique combination of symptoms and pathological hallmarks. A large body of research has shown that oligomeric tau is the most toxic form of the protein and is capable of seeding the spread of disease pathology throughout the brain. It has been hypothesized that these prion-like properties extend to the formation of conformationally distinct strains of many amyloidogenic proteins. Methods: We have isolated and characterized tau oligomeric strains from human disease tissues, including: Alzheimer's disease, progressive supranuclear palsy and Lewy body dementia and propagated them in hTau mice. Results: Systematic biochemical analyses suggest that these oligomers represent disease-specific tau oligomeric strains. When injected intravitreally in hTau mice, these oligomeric tau strains spread via the eye-brain axis and propagation led to diverse behavioral effects and seeding patterns. Conclusions: These findings suggest that differences in tau oligomeric strains can induce different phenotypes that are disease-specific, which has never been seen before. Additional in vitro studies suggest that direct interactions with other amyloidogenic proteins, such as $\mathrm{A} \beta$ and $\alpha$ synuclein, may play a role in the formation of distinct tau oligomeric strains. Further study of tau strains in terms of 1) both interand intra-disease strains, 2) their dynamic interaction between different strains and 3) their specific progression, pathology and phenotypes in neurodegenerative tauopathies need to be addressed. These studies could lead to new insights into previously unknown mechanisms of disease onset and diversity and improve investigation of potential biomarkers, imaging reagents and therapeutics. 